\title{
Momentum Transfer within Canopies
}

\author{
ChUiXIANG Yi \\ Queens College, City University of New York, Flushing, New York
}

(Manuscript received 12 December 2006, in final form 21 March 2007)

\begin{abstract}
To understand the basic characteristics of the observed S-shaped wind profile and the exponential flux profile within forest canopies, three hypotheses are postulated. The relationship between these fundamental profiles is well established by combining the postulated hypotheses with momentum equations. Robust agreements between theoretical predictions and observations indicate that the nature of momentum transfer within canopies can be well understood by combining the postulated hypotheses and momentum equations. The exponential Reynolds stress profiles were successfully predicted by the leaf area index (LAI) profile alone. The characteristics of the S-shaped wind profile were theoretically explained by the plant morphology and local drag coefficient distribution. Predictions of maximum drag coefficient were located around the maximum leaf area level for most forest canopies but lower than the maximum leaf area level for a corn canopy. A universal relationship of the Reynolds stress between the top and bottom of the canopy is predicted for all canopies. This universal relationship can be used to understand what percentage of the Reynolds stress at the top of canopy is absorbed by the whole canopy layer from the observed LAI values alone. All of these predictions are consistent with the conclusions from dimensional analysis and satisfy the continuity requirement of Reynolds stress, mean wind speed, and local drag coefficient at the top of canopy.
\end{abstract}

\section{Introduction}

The exchange of materials and energy between plant canopies and the atmosphere is the foundation of some of the most important environmental challenges facing humankind, including perturbations to the global carbon cycle, the introduction of pollutants into the atmosphere, and the transfer of water from soil and vegetation to the atmosphere (Schimel et al. 2001; Vitousek et al. 1997; Wofsy 2001). Turbulent transport processes that occur within canopies are extremely complex and have not been adequately represented in models, causing poor environmental analysis and prognosis (Massman and Weil 1999; Finnigan 2000).

Canopy turbulent flows are characterized by two fundamental profiles (Fig. 1): the S-shaped wind profile and the exponential Reynolds stress profile. The Sshaped wind profiles have been widely observed within forest canopies (Baldocchi and Meyers 1988; Bergen 1971; Fischenich 1996; Fons 1940; Lalic and Mihailovic

Corresponding author address: Chuixiang Yi, School of Earth and Environmental Sciences, Queens College, City University of New York, 65-30 Kissena Blvd., Flushing, NY 11367.

E-mail: cyi@qc.cuny.edu
2002; Landsberg and James 1971; Lemon et al. 1970; Meyers and Paw U 1986; Oliver 1971; Shaw 1977; Turnipseed et al. 2003; Yi et al. 2005). The S-shaped profile refers to a secondary wind maximum that is often observed within the trunk space of forests and a secondary minimum wind speed in the region of greatest foliage density. For crops or other more uniform plant canopies, the secondary wind maximum is very weak and observed wind speeds are almost constant in the lower part of canopy (Allen 1968; Legg and Long 1975; Uchijima and Wright 1964), as shown by the thin solid line in Fig. 1. Regardless of whether the vegetation is a forest or a crop, the Reynolds stress profiles within canopy always follow an exponential shape (Amiro 1990; Baldocchi and Meyers 1988; Katul and Albertson 1998; Katul et al. 2004; Kelliher et al. 1998; Shaw 1977; Wilson 1988).

The relationship between these fundamental profiles is key to understanding the transport dynamics of chemical compounds and reaction products within the canopy. The mixing length hypothesis, postulated by Prandtl to derive the logarithmic velocity profile from the constant flux profile above canopy, is not valid within canopy (Massman 1997; Raupach and Thom 1981). Yi et al. (2005) applied the relationship between the Reynolds stress and velocity squared, which has 


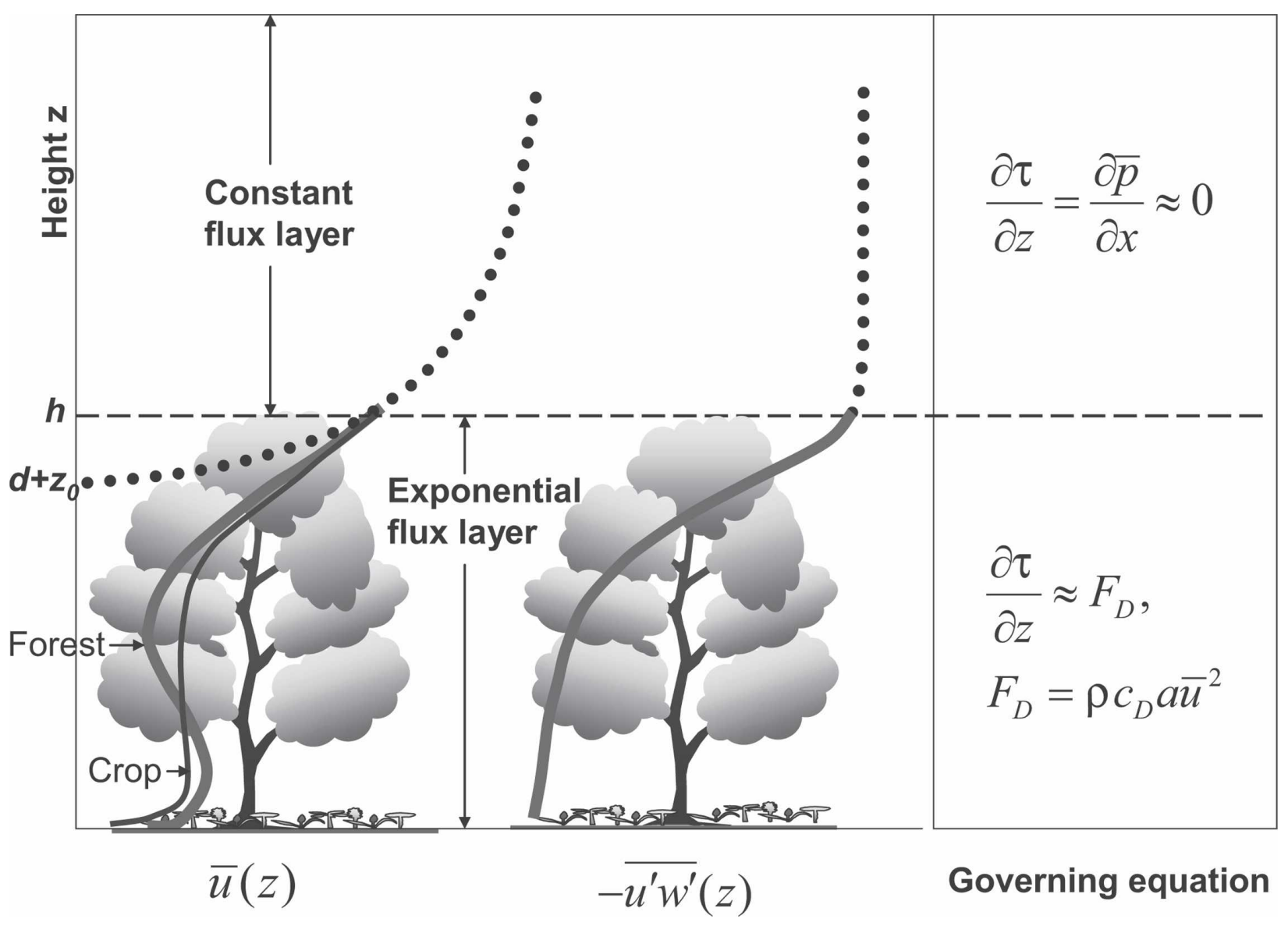

FIG. 1. Fundamental patterns of wind speed and the Reynolds stress within and above canopy and their governing equations.

been widely used in the constant flux layer over a rough surface or above canopy (Massman 1987; Mahrt et al. 2000; Monteith and Unsworth 1990; Raupach 1992; Sutton 1953), to within-canopy flow and established relationships between these fundamental profiles. However, Yi et al. (2005) did not adequately address the physics behind their formulation. Also, this new formulation has not been adequately validated by the experiments conducted at the Niwot Ridge AmeriFlux site since turbulent fluxes were measured only in the tree trunk space and above canopy and not in the upper canopy layer. In this paper, the formulation is rationalized into a theoretical framework, and the consistency between theoretical predictions and observations is tested utilizing previously published data (see appendix).

\section{Theoretical background}

This section addresses 1) the classic hypotheses and basic characteristics of turbulent flow above canopy; 2 ) why Prandtl's mixing length theory is successful in describing the basic characteristics of airflow near most natural surfaces, but not within canopy; 3) the limitation of the widely used, Inoue model; and 4) the weaknesses of the higher-order closure approach.

\section{a. The classic hypotheses and flow above canopy}

Most hypotheses postulated in boundary layer theory endeavor to establish a relationship between Reynolds stresses and mean velocity (Schlichting 1960). The main empirical hypotheses are summarized as follows:

$$
\frac{\tau}{\rho}=-\overline{u^{\prime} w^{\prime}}=u_{*}^{2}=\left\{\begin{array}{l}
K_{m} \frac{\partial \bar{u}}{\partial z}, \quad K \text { theory, proposed by Boussinesq in } 1877 \\
\ell^{2} \mid \frac{\partial \bar{u}}{\partial z} \frac{\partial \bar{u}}{\partial z}, \quad \text { mixing length theory, developed by Prandtl in 1925, and } \\
c_{D} \bar{u}^{2}, \quad \text { proposed by Prandtl in } 1932 \text { based on the velocity-squared law }
\end{array}\right.
$$


where $\tau$ is the turbulent shearing stress, $K_{m}$ is the eddy viscosity, $\ell$ is the mixing length, and $u_{*}$ is the friction velocity. The gradient transport hypothesis $(K$ theory) (1), initially proposed by Boussinesq (1877), could not be effectively used until Prandtl developed the mixing length hypothesis (Prandtl 1925). Although one unknown (mixing length) merely replaced another (eddy viscosity), by $K_{m}=\ell^{2}|\partial \bar{u} / \partial z|$, the introduction of the mixing length has led to vigorous studies both theoretically and experimentally. To determine mixing length, $\ell$, further plausible hypotheses are needed. Von Kármán proposed a similarity hypothesis in which mixing length satisfies the equation

$$
\ell=\kappa\left|\frac{d \bar{u} / d z}{d^{2} \bar{u} / d z^{2}}\right|,
$$

where $\kappa$ is an empirical constant. The logarithmic velocity profile is derived with the assumption of a linear shearing stress distribution from the surface (von Kármán 1930). Prandtl (1925) derived the logarithmic velocity profile, assuming that the mixing length was proportional to the distance from the surface, $\ell=\kappa z$, and that shearing stress remains constant. These two hypotheses lead to identical velocity profiles and to the establishment of the constant, $\kappa(\approx 0.4$, von Kármán constant). The logarithmic velocity distribution is found to apply to the flow near any rough surface. The empirical extension to very rough surface (tall vegetation) is written as

$$
\frac{\bar{u}}{u_{*}}=\frac{1}{\kappa} \ln \left(\frac{z-d}{z_{0}}\right), \quad z \geq h,
$$

where $d$ is a zero-plane displacement, $z_{0}$ is roughness height, and $h$ is canopy height. The most remarkable feature of the logarithmic velocity distribution is that it satisfies the velocity-squared law (3) (Brunt 1939, 259260; Oke 1987, p. 76; Schlichting 1960, p. 480; Sutton 1953, 256-257). Taylor (1916) was the first to test the validity of the velocity-squared law on the earth's surface and estimated its drag coefficient values. Numerous experiments conducted since Taylor's investigation demonstrate that the values of drag coefficients for different natural surfaces are of the same order of magnitude as those employed in aerodynamics (Deacon 1949; Mahrt et al. 2001; Sutcliffe 1936; Sutton 1953). These natural surface drag coefficient estimates are usually made during adiabatic conditions. Mahrt et al. (2001) examined the dependence of the drag coefficient on stability.

Airflow near a rough surface or above a canopy is characterized by the logarithmic velocity distribution and constant Reynolds stress (Fig. 1). The mixing length theory (or $K$ theory) has achieved remarkable success in describing a relationship between these two fundamental profiles. Since the logarithmic velocity distribution is derived based on the constant flux assumption, the logarithmic layer is consistent with the constant flux layer (Fig. 1). The constant flux assumption is based on the fact that the pressure gradient near a natural surface (or wall) is small. If the $x$ axis is in the direction of the mean wind, neglecting advection and the Coriolis force, the Reynolds stress is governed by

$$
\frac{\partial \tau}{\partial z}=\frac{\partial \bar{p}}{\partial x}
$$

where $\partial \bar{p} / \partial x$ is the pressure gradient in the direction of the mean wind and is assumed to be independent of height in the shallow layer above canopy. Integrating Eq. (6) from the top of canopy $h$ to level $z$,

$$
\tau(z)=\tau(h)+(z-h) \frac{\partial \bar{p}}{\partial x} .
$$

In most meteorological problems, $\tau_{h}=\tau(h) \gg(z-$ $h) \partial \bar{p} / \partial x$ (Sutton 1953), provided that $(z-h)$ is not large. Thus, $\tau_{h}=$ constant is a reasonable approximation (Wyngaard 1973). The friction velocity, $u_{*}=$ $\sqrt{\tau_{h} / \rho}=\sqrt{\left|u^{\prime} w^{\prime}\right|}$, was initially introduced as an auxiliary reference velocity and is constant in the logarithmic layer. Sutton (1953) commented, "the friction velocity is the artificial but related velocity for which the square law holds exactly." Obviously, the square law (3) can be derived from the logarithmic velocity distribution. For statically nonneutral conditions, a stability correction factor can be included in Eq. (5) (Stull 1988). These stability correction factors are related to the Monin-Obukhov similarity theory, which is valid in the same layer as the logarithmic law (Obukhov and Monin 1953).

\section{b. The mixing length theory and flow within a canopy}

Many attempts have been made to apply mixing length theory to within-canopy flow to interpret the basic characteristics of canopy turbulent flow by empirical modifications of the logarithmic velocity distribution (5) (Barr 1971; Cionco 1965; Inoue 1963; Jackson 1981; Macdonald 2000; Uchijima and Wright 1964). However, all the extensions and applications of mixing length theory to within-canopy flows have never been successful in describing the S-shaped wind profile and the exponential flux profile (Raupach and Thom 1981).

Theoretically, mixing length theory is unable to describe the S-shaped wind profile for two reasons. First, 
mixing length vanishes at the level of the secondary wind speed maximum and at the level of minimum wind speed in the upper part of the canopy. This is a necessary because of von Kármán's condition in Eq. (4); otherwise turbulence would be nonexistent at the locations. Second, the negative wind gradient $\partial \bar{u} / \partial z$ in the lower part of the canopy would lead to a negative eddy viscosity $K_{m}$ as predicted by Eq. (1), based on the fact that the momentum flux throughout the canopy must be downward. This is a well-known problem with countergradient momentum transport. Similarly, many observations have demonstrated that all gradientdiffusion schemes including momentum, mass, and heat fail completely within canopy (Denmead and Bradley 1985).

\section{c. The spatial-average scheme and simplified governing equation}

As discussed above, the mixing length $\ell$ is not appropriate to use as the length scale within canopy. The drag exerted on flow by plant elements is an essential part of understanding air-plant interactions. Thom (1968) tested all aspects of the drag coefficient of a single artificial leaf with wind tunnel experiments. However, the drag forces within plant canopies are far more complex than a single leaf. Complex plant canopy structures not only create interference drag (shelter effect; Massman 1997), they also generate three-dimensional turbulent structures (Raupach and Thom 1981).

The spatial averaging scheme (hereinafter referred to as WSRF averaging scheme) was a revolutionary step to simplify three-dimensional canopy flow into a onedimensional description. WSRF was first introduced by Wilson and Shaw (1977) and further developed by Raupach and Shaw (1982) as an area average over a horizontal plane intersecting numerous plants. The more general volume averaging approach was subsequently developed by Finnigan (1985) and Raupach et al. (1986). The choice of averaging volume is usually a thin, wide, horizontal slab that preserves the fundamental vertical heterogeneity of the canopy but reflects its horizontal uniformity on the scale of many plants. More details about the WSRF averaging scheme can be found in Raupach and Thom (1981). The simplified governing equation is

$$
\frac{\partial \tau}{\partial z}=\rho c_{D}(z) a(z) \bar{u}^{2}(z)=F_{D}(z),
$$

where $F_{D}(z)$ is a drag force that results physically from the noncommutative nature between the spatial averaging and differentiation (in contrast to adding the drag forces arbitrarily into the equation of motion as before), $a(z)$ is leaf area density (frontal area per unit volume), and $c_{D}(z)=C_{D}(z) / p_{M}$ is an effective drag coefficient where $p_{M}$ is a shelter factor (Massman 1997). For convenience, the symbols $\tau(z)=-\rho \overline{u^{\prime} w^{\prime}}(z)$ and $\bar{u}(z)$ are used to denote the WSRF average variables.

The steady-state Eq. (8) is valid for horizontally homogeneous, plane turbulence with no static pressure gradient, advection, Coriolis force, and dispersion of flux terms. With the exception of the dispersive flux terms, these simplifications are typically assumed. The dispersive fluxes result from the spatial correlation of regions of mean updraft or downdraft with regions where $\bar{u}(z)$ differs from its spatial mean (Raupach and Thom 1981). Wind tunnel experiments demonstrate that the dispersive fluxes can be neglected in dense canopies across the entire depth of canopy (Poggi et al. 2004a; Cheng and Castro 2002; Raupach et al. 1986). However, for sparse canopies, the dispersive fluxes can be large in the bottom layers of the canopy (Poggi et al. 2004b; Bohm et al. 2000). These wind tunnel observations are supported by the experiments conducted in real forests, which show that the dispersive fluxes are typically on the order of $0 \%-20 \%$ (on average $14 \%$ ) of the mean momentum flux within the lower layer of the sparse canopy (Christen and Vogt 2004). Therefore, the derivations from Eq. (8) will be valid for dense canopies, and subject to a dispersive flux correction in the bottom layers of sparse canopies.

\section{d. Inoue's analytical solution}

Inoue (1963) found an analytical solution for Eq. (8) based on these assumptions: 1) the vertical leaf area distribution is uniform, $a(z)=a$;2) the drag coefficient is constant, $c_{D}(z)=c_{D}$; and 3 ) the mixing length is constant with height except very near the ground. Inoue first assumed the solution of (8) in exponential form:

$$
\bar{u}(z)=\bar{u}_{h} e^{\alpha[(z / h)-1]},
$$

where $\bar{u}_{h}$ is the wind speed at the top of the canopy, and then used the above three assumptions to determine the attenuation coefficient, $\alpha=\beta h / \ell$ [where $\beta=u_{*} / \bar{u}_{h}$, $\ell=2 \beta^{3} L_{c}$, and $\left.L_{c}=\left(c_{D} a\right)^{-1}\right]$ from Eq. (8) with empirical adjustments (Cionco 1965). This simple solution has been widely used for crop canopies (Raupach and Thom 1981) and urban canopies of buildings (Belcher et al. 2003), where the drag elements have a uniform vertical distribution. The upper part of most canopy profiles can be well described by the exponential profile (9), with a reasonable choice of $\alpha$ values. This portion of the canopy is sometimes called the shear layer within which the constant mixing length assumption is likely 
valid. However, the mixing length concept cannot be used in regions where the wind profile reaches a minimum or maximum, or where it remains constant with height because the mixing length is zero as discussed above, according to von Kármán's similarity law in Eq. (4).

\section{e. Higher-order closures}

Attempts to interpret the S-shaped wind profile by modifying the exponential profile in Eq. (9), have not been successful because of the intrinsic weaknesses discussed above (Albini 1981; Mohan and Tiwari 2004). To interpret the basic characteristics of within-canopy flow, several higher-order closure models have been developed (Katul and Albertson 1998; Katul and Chang 1999; Meyers and Paw U 1986; Wilson 1988). Wilson and Shaw (1977) first proposed the secondorder closure scheme for within-canopy flow. The basic characteristics of within-canopy flow can be successfully simulated by these numerical, higher-order closure models. However, the use of numerous adjustable constants is a weakness of higher-order closure models, especially when treating the drag coefficient as an adjustable constant. The problem of treating the drag coefficient as an adjustable constant throughout the canopy layer has been recognized by several investigators (Ayotte et al. 1999; Brunet et al. 1994; Novak et al. 2000; Pinard and Wilson 2001; Poggi et al. 2004X). Even for a rodlike canopy, the drag coefficient exhibits a characteristic height dependence, as shown in wind tunnel experiments (Brunet et al. 1994).

\section{New hypotheses}

The basic physical processes in the lowest atmospheric layers, whether over bare ground or tall vegetation, are the downward transport processes of momentum and the dissipative processes of turbulent energy cascades. Drag is essential in this process and is generated when a fluid moves over the ground or through vegetation. Drag creates velocity gradients and eddies, characterized by the profiles of flow velocity and Reynolds stress (Fig. 1), which lead to momentum loss of the fluid. Information about downward momentum transfer is contained in the wind and Reynolds stress profiles, which are related to one another. The relationship between the two fundamental profiles cannot be derived from first principles but can be tested by semiempirical hypotheses, such as the $K$ theory expressed by Eq. (1), the mixing length theory in Eq. (2), and the velocity-squared law in Eq. (3).

In the case of bare ground, these semiempirical theories not only universally express the mathematical re- lationship between the logarithmic wind speed distribution and the constant Reynolds stress, but also physically explain the mechanisms of downward transport of horizontal momentum by the mixing length and friction velocity. This is because bare ground provides similar drag conditions and slows down wind in a shallow layer near the ground. However, for a vegetative canopy, complex canopy structures form an addition buffer layer over the ground in which 1) large-scale eddies are broken into smaller-scale eddies in the wake formed behind obstructions and 2) momentum is absorbed by canopy elements. Since mixing length theory failed to describe the basic characteristics of within-canopy flows as discussed previously, new hypotheses are proposed to establish the relationship between the mean wind speed and Reynolds stress. The new relationships will be used to formulate a closure approach for the momentum equations. With these hypotheses, we can predict the basic characteristics of within-canopy flows.

Consider a steady, two-dimensional mean flow (here the mean flow refers to the WSRF average flow), where $\bar{u}(z)$ denotes the mean wind speed in the direction of the streamline (i.e., $x$ axis), $\tau=-\rho \overline{u^{\prime} w^{\prime}}(z)$ is Reynolds stress, $\rho$ is air density, and the $z$ axis is normal to the ground. Assume that canopy elements are horizontally homogeneous with a continuous vertical distribution of leaf area density, $a(z)$, and that $h$ denotes the vegetation height.

\section{a. Hypothesis 1}

Within the canopy, the transport of horizontal momentum is continuous and downward. Meanwhile the horizontal momentum is continuously absorbed by canopy elements from the air.

\section{b. Hypothesis 2}

A local equilibrium exists between the rate of horizontal momentum transfer and its rate of loss. With appropriate averaging scales of time and space, the local equilibrium relationship at level $z$ is

$$
-\rho \overline{u^{\prime} w^{\prime}}(z)=\rho c_{D}(z) \bar{u}^{2}(z),
$$

where $c_{D}(z)$ is a drag coefficient (the factor one-half is absorbed in $c_{D}$ following the micrometeorological convention). To understand the physical meaning of the drag coefficient in Eq. (10), consider an extreme case where a fluid is uniformly decelerated from speed $\bar{u}$ to rest on the drag elements. If the initial momentum per unit volume of fluid is $\rho \bar{u}$ and the averaged wind speed during deceleration is $\bar{u} / 2$, the rate at which momentum is lost from the fluid is $\rho \bar{u} \times \bar{u} / 2=\rho \bar{u}^{2} / 2$. In practice, fluid tends to slip around the drag elements so that the 
momentum losses are less than $\rho \bar{u}^{2} / 2$ (Monteith and Unsworth 1990). Therefore, the physical meaning of $c_{D}(z)$ is the effectiveness of canopy drag elements in absorbing momentum from the airflow.

\section{c. Hypothesis 3}

The drag coefficient, $c_{D}(z)$, defined in Eq. (10), is equal to that defined in the volumetric drag force in the momentum equation if their averaging operations are same. The $c_{D}(z)$ can be determined empirically, and directly, from observed profiles of wind speed and Reynolds stress; $c_{D}(z)$ can be also deduced from theoretical predictions by substituting Eq. (10) into the momentum equation. Results from these two methods can be used to examine the consistency.

Equation (10) is consistent with dimensional analysis using the Buckingham pi theorem. The pertinent variables and their physical dimensions (listed below them) are posed as follows:

$$
\left\{\begin{array}{cccccc}
\tau & \rho & \bar{u} & \mu & h & a \\
m \ell^{-1} t^{-2} & m \ell^{-3} & \ell t^{-1} & m \ell^{-1} t^{-1} & \ell & \ell^{-1}
\end{array}\right\},
$$

where $\mu$ is the dynamic viscosity. Assume that Reynolds stress $\tau$ is a function of the remaining five variables in Eq. (11):

$$
\tau=f(\rho, \bar{u}, \mu, h, a) .
$$

Equation (12) can be written as

$$
g(\tau, \rho, \bar{u}, \mu, h, a)=0 .
$$

According to the Buckingham pi theorem, three dimensionless variables are necessary in Eq. (13). After simple manipulation, Eq. (13) becomes

$$
g\left(\frac{\tau}{\rho \bar{u}^{2}}, \frac{\rho \bar{u} h}{\mu}, a h\right)=0,
$$

or

$$
\tau=f_{1}(\mathrm{Re}, \mathrm{LAI}) \rho \bar{u}^{2},
$$

where $\operatorname{Re}=\rho \bar{u} h / \mu$ is the Reynolds number and $a h$ is a cumulative leaf area here replaced by leaf area index (LAI). Combining Eqs. (15) and (10),

$$
c_{D}=f_{1}(\mathrm{Re}, \mathrm{LAI}) \text {. }
$$

In calm atmospheric conditions (low wind speed), it is possible for $c_{D}$ to be a function of the Reynolds number (Grant 1983; Landsberg and Powell 1973; Maheshwari 1992; Mahrt et al. 2001; Murota et al. 1984; Schuepp 1984; Thom 1971), but in most meteorological conditions, $c_{D}$ is independent of the Reynolds number. Nevertheless, the canopy drag coefficient always depends on the LAI profile as demonstrated in the following sections.

Equation (10) is the extension of the universal velocity-squared law to within-canopy flows, where a local equilibrium is rapidly established between the downward transfer rate and rate of horizontal momentum loss. The above hypotheses are semiempirical and independent of the Navier-Stokes equations, but can be combined with the Navier-Stokes equations to predict the regimes of canopy turbulent motion. The validity of the above hypotheses can be judged by ascertaining if the consequences are consistent with observations. Several ways to test the above hypotheses are 1) the hypotheses should be consistent with dimensional analysis as discussed above; 2) the observed basic characteristics of the S-shaped wind profile and the exponential Reynolds stress profile can be predicted based on the hypotheses; 3) the values of the local drag coefficient, determined empirically by Eq. (10), should be consistent with theoretical predictions; 4) the new (proposed) theory does not contradict the existing theory but includes it as a specific case; and 5) all theoretical predictions deduced from the above hypotheses must satisfy the continuity requirements of the variables such as wind speed $\bar{u}$, Reynolds stress $\tau$, and the drag coefficient $c_{D}$ at the top of the canopy, where they become bulk variables of a unit equivalent column of vegetation.

According to the above hypotheses, Eq. (8) is easily closed, provided that both Eqs. (8) and (10) are averaged by the same WSRF averaging operations. With these hypotheses, Eq. (8) becomes a solvable equation either for the mean wind speed

$$
\frac{d\left[c_{D}(z) \bar{u}^{2}(z)\right]}{d z}=a(z) c_{D}(z) \bar{u}^{2}(z)
$$

or for the Reynolds stress

$$
-\frac{d \overline{u^{\prime} w^{\prime}}(z)}{d z}+a(z) \overline{u^{\prime} w^{\prime}}(z)=0 .
$$

To understand what causes change in the momentum loss rate (or momentum transfer rate), $p(z)=$ $\rho c_{D}(z) \bar{u}^{2}(z)$, integrate Eq. (17) from $z_{1}$ to $z_{2}$. We obtain

$$
p\left(z_{2}\right)-p\left(z_{1}\right)=\int_{z_{1}}^{z_{2}} F_{D}\left(z^{\prime}\right) d z^{\prime},
$$

where $0 \leq z_{1}<z_{2} \leq h$, and $F_{D}(z)=\rho a(z) c_{D}(z) \bar{u}^{2}(z)$. The implication of Eq. (19) is that the reduction of horizontal momentum loss downward from $z_{2}$ to $z_{1}$ is uniquely determined by the total integrated drag force exerted on the airflow by the drag elements of unit 
equivalent column of the layer $\Delta z=z_{2}-z_{1}$. The implication is the same for the momentum transfer rate.

\section{Inoue's model as a trial solution}

Assume that the vegetation has a uniform vertical distribution of leaf area $[a(z)=a=$ constant $]$ and drag coefficient $\left[c_{D}(z)=c_{D}=\right.$ constant $]$. Equation (17) becomes

$$
\frac{d q(z)}{d z}=a q(z)
$$

where $q(z)=c_{D} \bar{u}^{2}(z)$. Integrating Eq. (20) from $z$ to the top of canopy,

$$
q(z)=q(h) \exp [a(z-h)]=q(h) \exp [\operatorname{LAI}(z / h-1)],
$$

where $q(h)=c_{D} \bar{u}_{h}^{2}$, LAI $=a h$, and $\bar{u}_{h}$ is mean wind speed at the top of the canopy. Using $q(z)=c_{D} \bar{u}^{2}(z)$, the solution of Eq. (21) can be written as

$$
\bar{u}(z)=\bar{u}_{h} \exp \left[\frac{\mathrm{LAI}}{2}\left(\frac{z}{h}-1\right)\right] .
$$

Inoue's model defines the attenuation coefficient $\alpha$ in (9) as

$$
\alpha=\frac{\beta h}{l}=\frac{\beta h}{2 \beta^{3} L_{c}}=\frac{h c_{D} a}{2 \beta^{2}}=\frac{a h}{2}=\frac{\mathrm{LAI}}{2},
$$

where $c_{D}=\beta^{2}=\left(u_{*} / u_{h}\right)^{2}$ is used.

Inoue's model was derived without using mixing length theory and the attenuation coefficient in this model was derived as one-half LAI. Many observations show that the attenuation coefficient $\alpha$ is related to the LAI (Cionco 1972; Jackson 1981; Macdonald 2000). Combining Eqs. (10) and (21), the Reynolds stress is predicted as

$$
\tau(z)=\tau_{h} \exp [\operatorname{LAI}(z / h-1)],
$$

where $\tau_{h}$ is the Reynolds stress at the top of canopy. The LAI dependence of the Reynolds stress $\tau(z)$ is consistent with the conclusion [Eq. (15)] of the dimensional analysis. The normalized form of Eq. (24) is

$$
\tilde{\tau}=e^{\mathrm{LAI}(\zeta-1)},
$$

where $\tilde{\tau}=\tau(z) / \tau_{h}$ and $\zeta=z / h$. This result indicates that the normalization Reynolds stress profiles of all vertically uniform canopies collapse into a unique curve that depends only on LAI (Fig. 2).

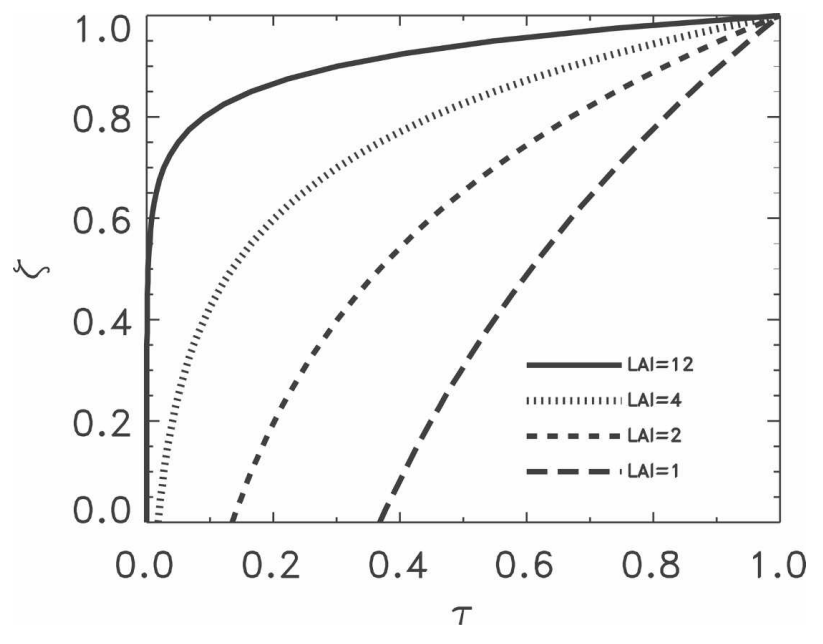

FIG. 2. The universal distribution of the normalization Reynolds stress [Eq. (25)] for all uniform canopies. The horizontal axis is normalized Reynolds stress and the vertical axis is normalized height.

\section{Predictions and observations}

For most vegetation canopies, particularly forest canopies, the variation in vertical leaf area distribution is large. In this section, theoretical predictions are compared with the observations using previously published data for canopies with nonuniform vertical leaf area distributions.

\section{a. Exponential Reynolds stress profile}

If the cumulative leaf area per unit ground area below height $z$ is defined as

$$
L(z)=\int_{0}^{z} a\left(z^{\prime}\right) d z^{\prime},
$$

the solution of Eq. (18) is obtained as

$$
-\overline{u^{\prime} w^{\prime}}(z) / u_{*}^{2}(h)=\exp \{-[\mathrm{LAI}-L(z)]\}
$$

with the top boundary condition, where LAI $=L(h)$, and $u_{*}^{2}(h)=-\overline{u^{\prime} w^{\prime}}(h)$ is the Reynolds stress at the top of the canopy, or

$$
-\overline{u^{\prime} w^{\prime}}(z)=-\overline{u^{\prime} w^{\prime}}(0) e^{L(z)}
$$

where $-\overline{u^{\prime} w^{\prime}}(0)$ is Reynolds stress at the bottom of the canopy. The boundary conditions for Reynolds stress at the bottom and top of a canopy are related to one another as

$$
-\overline{u^{\prime} w^{\prime}}(h)=-\overline{u^{\prime} w^{\prime}}(0) e^{\mathrm{LAI}}
$$

or

$$
\tau_{0} / \tau_{h}=e^{-\mathrm{LAI}}
$$

where $\tau_{0}=-\overline{u^{\prime} w^{\prime}}(0)$ and $\tau_{h}=-\overline{u^{\prime} w^{\prime}}(h)$. This simple relationship is universal to all canopies. As shown in 


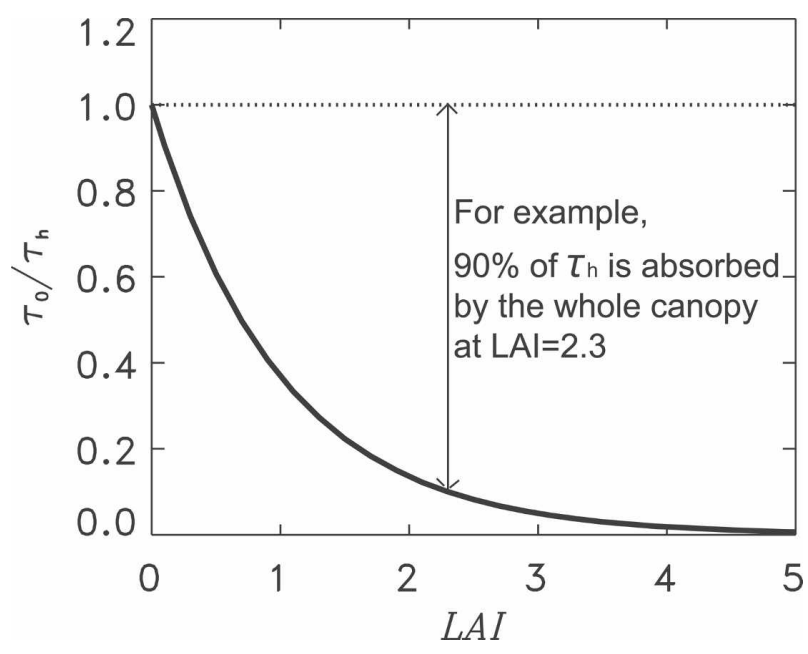

FIG. 3. The relationship between the ratio of the Reynolds stress at the bottom and top of canopy and LAI. Here $\tau_{0}=-\overline{u^{\prime} w^{\prime}}(0)$ denotes Reynolds stress at the ground, and $\tau_{h}=-\overline{u^{\prime} w^{\prime}}(h)$ is Reynolds stress at the top of canopy.

Fig. 3, a unique ratio of the Reynolds stress at the bottom and the top of the canopy versus LAI is predicted by Eq. (29). The physical meaning of this universal curve is that the LAI determines what percentage of horizontal momentum at the top of canopy is absorbed by the whole canopy. When the LAI reaches about 5 , almost all of the momentum at the top of the canopy is absorbed by canopy elements.

The solutions in both Eqs. (27) and (28) indicate that the downward transfer rate of momentum at $z$ is uniquely determined by the cumulative leaf area of a unit equivalent column between $z$ and some reference level [i.e., LAI $-L(z)$ in Eq. (27) is the cumulative leaf area between $z$ and the top of canopy, and $L(z)$ is the cumulative leaf area between the ground and $z$ ]. Again, the relationship between the Reynolds stress and the cumulative leaf area is also consistent with the predictions from dimensional analysis.

The predictive ability of Eq. (27) can be tested using previously published data on leaf area density and normalized Reynolds stress (Table 1; appendix). The calculated Reynolds stress profiles from Eq. (27), using measured leaf area density profiles as input, accurately describe empirically determined patterns (Fig. 4).

The comparisons of predictions between the present model and two higher-order closure models (Wilson and Shaw 1977; Albini 1981) are also illustrated in Fig. $4 \mathrm{a}$. The observed leaf area density data reported by Shaw (1977) were used in the three models for this comparison. The theoretical prediction of the Reynolds stress profile is equally as good, or better, than the more complicated higher-order closure models. How-
TABLE 1. Canopy morphology. COa and COb are different corn canopies, AS is the aspen stand, HW is the hardwood forest, JPI is the jack pine stand, LPI is the loblolly pine stand, SP is the spruce stand, and SPI is the Scots pine stand. Details of sites and measurements are described in the appendix.

\begin{tabular}{lcccccccc}
\hline \multicolumn{1}{c}{ Canopy } & COa & COb & AS & HW & JPI & LPI & SP & SPI \\
\hline$h(\mathrm{~m})$ & 2.9 & 2.2 & 10 & 22 & 15 & 16 & 10 & 20 \\
LAI $\left(\mathrm{m}^{2} \mathrm{~m}^{-2}\right)$ & 3.0 & 2.9 & 4.0 & 5.0 & 2.0 & 3.8 & 10.0 & 2.6 \\
\hline
\end{tabular}

ever, the theoretical prediction in Eq. (27) or (28) is determined by the LAI profile alone and the drag coefficient information is not necessary, while the higherorder closure models use several adjustable constants and treat the drag coefficient as a free parameter.

\section{b. The S-shaped wind profile}

The mean wind speed can be obtained by directly integrating Eq. (17), or by substituting Eq. (10) into (27); thus,

$$
\bar{u}(z)=\bar{u}_{h}\left[c_{D}^{h} / c_{D}(z)\right]^{1 / 2} \exp \{-0.5[\mathrm{LAI}-L(z)]\},
$$

where $c_{D}^{h}$ is the drag coefficient at the top of canopy. Equation (30) successfully explains why the secondary wind speed maximum is often observed in the trunk space within a forest canopy, while the minimum wind speed is located around the maximum canopy drag level.

The prediction of minimum wind speed is important because Yi et al. (2005) found that a superstable layer was formed around the maximum canopy drag level if the potential temperature gradient is significant. The superstable layer is characterized by 1) slow mean airflow; 2) maximum density of drag elements; 3) nearzero vertical velocity; 4) minimum vertical exchange of mass or energy; 5) maximum horizontal $\mathrm{CO}_{2}$ gradient (or other scalar gradient; Yi et al. 2008); and 6) maximum ratio of wake and shear production rate (Yi et al. 2005). The existence of the superstable layer was tested by conducting $\mathrm{SF}_{6}$ diffusion experiments with a fourtower system at the Niwot Ridge AmeriFlux site in the Rocky Mountains of Colorado (Yi et al. 2005).

The existence of the superstable layer can be viewed as a condition of airflow separation at night. A vegetation canopy can be divided into two regions: the region above the superstable layer (the "vertical exchange zone") where vertical turbulent mixing dominates, and the region below the superstable layer (the "longitudinal exchange zone") where horizontal advection prevails. The superstable layer creates difficult conditions for eddy flux measurement of $\mathrm{CO}_{2}$ at night. The theoretical explanations of the S-shaped wind profile char- 

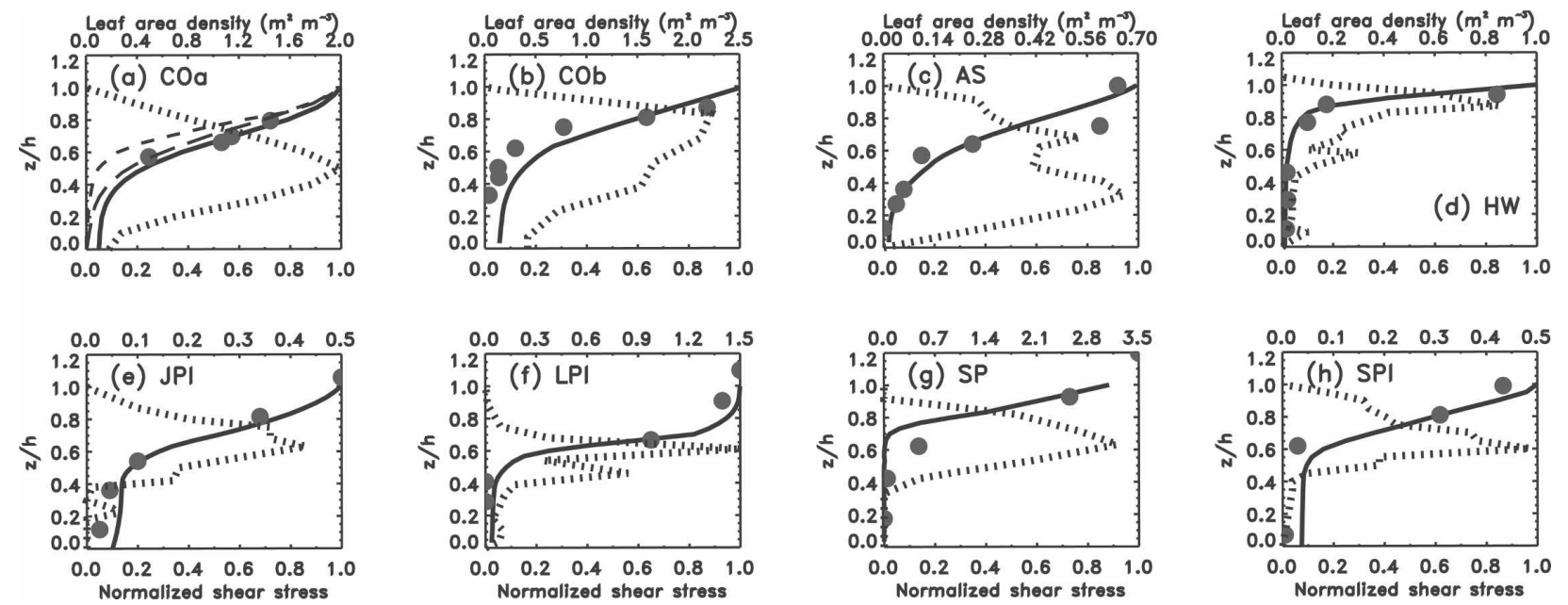

FIG. 4. Comparison of predicted normalization Reynolds stress profiles from the present model (solid line) with observed data (filled circle) in eight vegetation types (see Table 1) reported in the literature (Shaw 1977; Katul and Albertson 1998; Katul et al. 2004; Wilson 1988; Amiro 1990; Baldocchi and Meyers 1988; Kelliher et al. 1998). The comparison of the prediction between the present model and the higher-order closure models is also shown in (a): the long dashed line was predicted by Wilson and Shaw's model (Wilson and Shaw 1977), and the dashed line was predicted by Albini's model (Albini 1981).

acteristics are helpful in understanding the nighttime advective flux, of interest to the eddy flux measurement community (Yi et al. 2008).

To summarize, good agreement is evident between the predicted wind speed by Eq. (30) and the observations based on the previously published data (Fig. 5; Table 1; appendix). The drag coefficients were calculated by Eq. (10) from the measurements of wind speed and Reynolds stress. The cumulative leaf area function $L(z)$ was calculated from the measured leaf area density profiles shown in Fig. 4. Therefore, the drag coefficient profile is needed to predict the wind speed profile. For this reason the prediction of wind speed has phenomenological features. Here the drag coefficient profile is measurable, but not adjustable, distinguishing the present approach from that of higher-order closure models.

\section{c. The canopy drag coefficient}

The canopy drag coefficient profile can also be predicted by the solution of the momentum Eq. (17). To understand the profile similarity between canopy structure, wind speed, and drag coefficient described in Eq. (17), the solution is written in nondimensional form

$$
\varphi_{c_{D}}(z) \varphi_{\bar{u}}^{2}(z)=e^{-\operatorname{LAI}[1-\xi(z)]}
$$

where $\varphi_{c_{D}}(z)=c_{D}(z) / c_{D}^{h}, \varphi_{\bar{u}}(z)=\bar{u}(z) / \bar{u}_{h}$, and $\xi(z)=$ $L(z) /$ LAI. The solution in Eq. (31) implies that if the normalization profiles of wind speed $\varphi_{\bar{u}}(z)$ and cumulative leaf area $\xi(z)$ are the same between two systems, the relative distribution of the drag coefficient, $\varphi_{c_{D}}(z)$, differs only by LAI. This implication is once again consistent with dimensional analysis (16).

The agreement between model predictions and observations of the local canopy drag coefficients are shown in Fig. 6. The magnitudes of the local drag coefficients observed and predicted here are within the ranges observed by wind tunnel experiments for terrestrial canopies (0-2) (Brunet et al. 1994). Maximum drag coefficients are located around the maximum leaf area density levels for most forest canopies, but for a corn canopy, the level $(z / h=0.45)$ with the maximum drag coefficient is lower than the level $(z / h=0.8)$ of maximum leaf area density (Fig. 6). The inconsistent location between the maximum drag coefficient and maximum leaf area density is possibly caused by the stronger bending effect of the corn canopy relative to forest canopies. The drag coefficient at $z$ predicted by Eq. (31) is related to the total integrated leaf area of a unit equivalent column between $z$ and the top of canopy rather than leaf area at $z$ alone. Actually, the canopy drag coefficient at $z$ depends on a total leaf area of a unit column between $z$ and any reference level where the drag coefficient is known. For example, if the bottom of a canopy is chosen as a reference level, the prediction equation of canopy drag coefficient becomes

$$
c_{D}(z)=c_{D}^{0} \frac{\bar{u}_{0}^{2}}{\bar{u}^{2}(z)} e^{L(z)},
$$

where $c_{D}^{0}$ and $\bar{u}_{0}$ are, respectively, drag coefficient and wind speed at the ground. In such a condition, the drag coefficient at $z$ is related to the integrated leaf area 

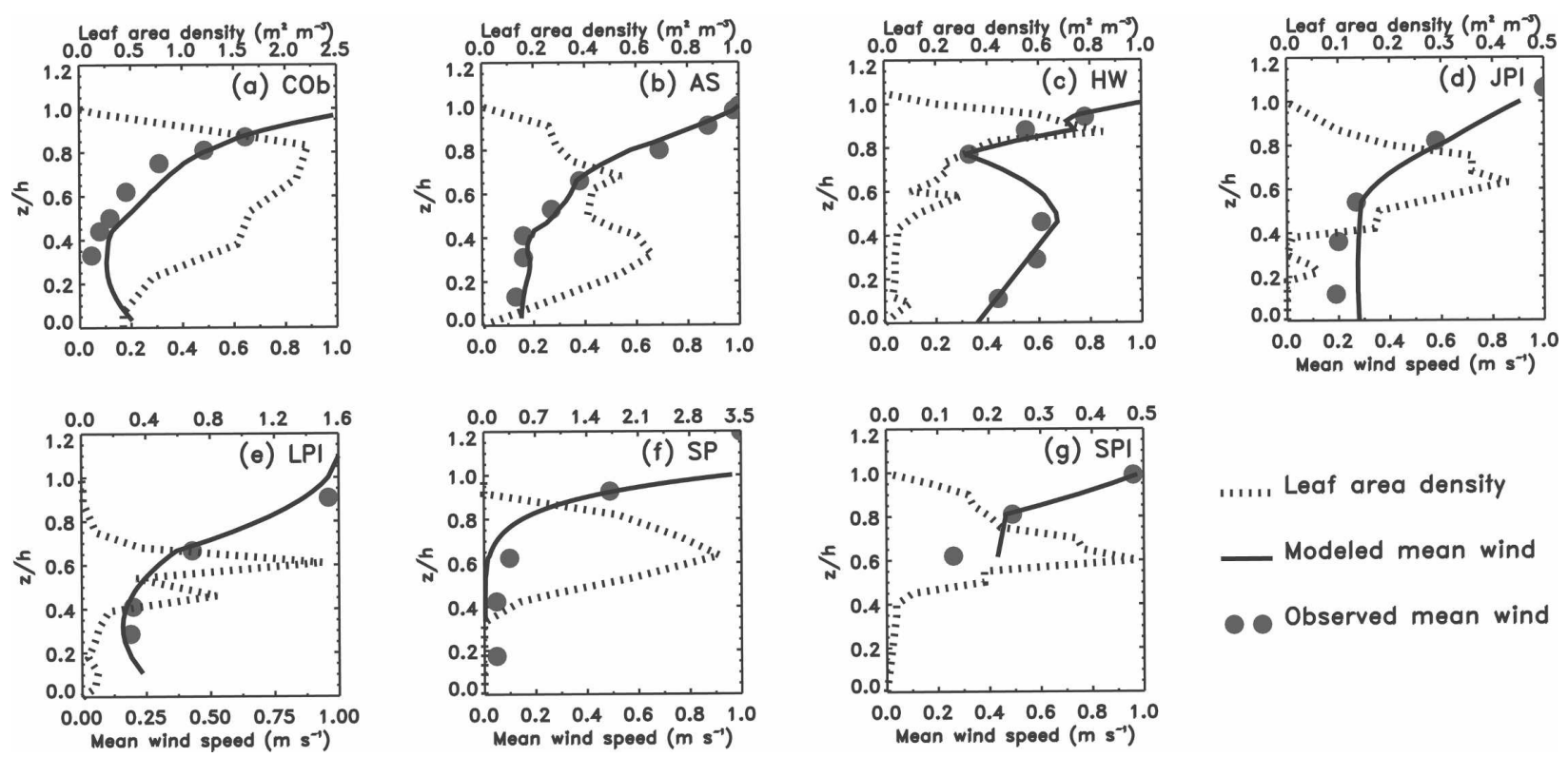

i..., Leaf area density

Modeled mean wind

Observed mean wind

FIG. 5. Comparison between present model predictions and observations across seven vegetation types for wind speed. The symbols indicate vegetation types as listed in Table 1. The absence of COa data that are in Figs. 4 and 5 is due to the fact that measured wind speeds and Reynolds stresses were not at the same levels (see Shaw 1977, his Figs. 1 and 2).

from the ground to $z$. In practice, however, measuring the drag coefficient at the ground is far more difficult than at the top of canopy. Therefore, the top boundary conditions are often used in practice. However, the bottom boundary condition is related to the top boundary condition by the relation

$$
\frac{c_{D}^{0}}{c_{D}^{h}}=\frac{e^{-\mathrm{LAI}}}{\bar{u}_{0}^{2} \bar{u}_{h}^{2}} .
$$
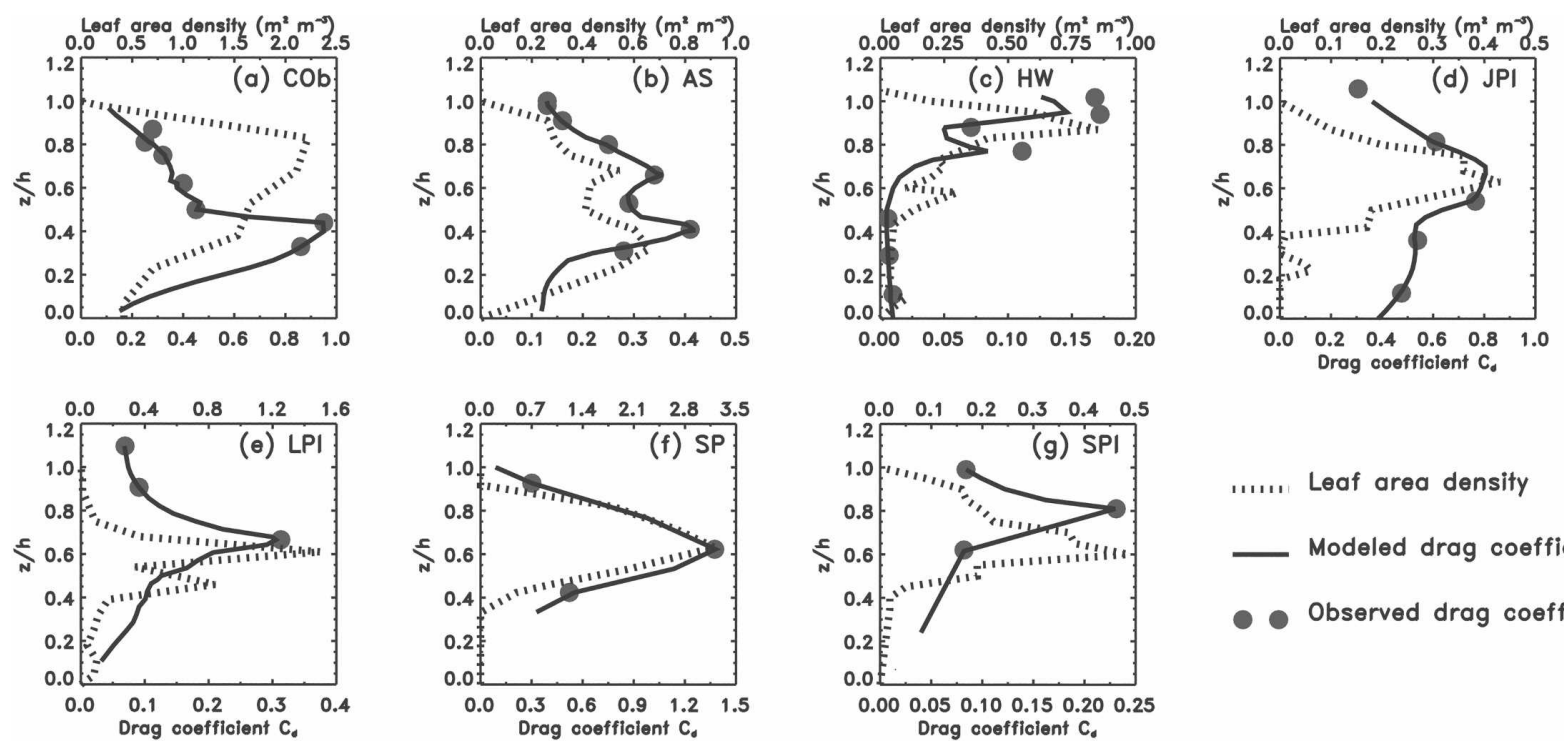

FIG. 6. Comparison of predicted drag coefficient profiles from the present model (solid line) to observed data (same sources as Fig. 5). The observed drag coefficients were calculated by Eq. (10) from the observed data of wind speed and Reynolds stress.
Equations (27), (30), and (31) indicate that all derivations from the posed hypotheses satisfy the continuity requirement of $\tau, \bar{u}$, and $c_{D}$ at the top of canopy.

\section{Summary and concluding remarks}

The hypotheses postulated in this paper can be summarized briefly as follows: 1) within the canopy, the transport and loss of horizontal momentum is continu- 
ous and downward; 2) a local momentum transfer rate, $-\rho \overline{u^{\prime} w^{\prime}}(z)$, is balanced by the rate of local momentum loss, $c_{D}(z) \bar{u}^{2}(z)$, provided that the average scales of time and space are appropriate; and 3) the drag coefficient, $c_{D}(z)$, is equivalent whether defined in the local equilibrium relationship or defined in the volumetric drag force in the momentum equations, if their averaging operations are the same. The validity of these hypotheses was tested against the observed data and previously published observations.

The momentum equations are closed using these hypotheses, and turbulence profiles of momentum are predicted. The model predictions were realistic and satisfactory when tested against the observed data of eight morphologically distinct canopies. The characteristics of the S-shaped wind profile were theoretically explained by the impact of plant morphology on the local drag coefficient distribution. The agreement between the predicted and observed wind speed was remarkably good. Predictions of maximum drag coefficient were located around the maximum leaf area level for most forest canopies but lower than the maximum leaf area level for a corn canopy. The widely used model of Inoue (1963) was derived without using mixing length theory, provided that the leaf area density and drag coefficient are constant in the vertical. A universal relationship of the Reynolds stress between the top and bottom of the canopy is predicted for all canopies. This relationship can be used to understand what percentage of the Reynolds stress at the top of canopy is absorbed by the entire canopy layer from the observed LAI values alone. All of these predictions are consistent with dimensional analysis and satisfy the continuity requirement of the Reynolds stress, mean wind speed, and local drag coefficient at the top of the canopy.

The most interesting prediction is that the Reynolds stress is uniquely determined by the LAI profile alone [see Eqs. (27) or (28)]. The prediction of the Reynolds stress is theoretical rather than phenomenological because the leaf area density profile (model input) can be directly measured. However, the predictions of the wind speed and drag coefficient profiles are still phenomenological. The prediction of the wind speed from Eq. (30) requires a known leaf area density distribution as well as the drag coefficient distribution [determined from Eq. (10) and previous wind speed observations]. Although the predictions of the wind speed have phenomenological features, these predictions have several advantages over higher-order closure models: 1) the canopy drag coefficient is determined by Eq. (10) from the observed wind speed and predicted Reynolds stress from the measured LAI profile (rather than treated as an adjustable constant); 2) no adjustable constants are used in the model predictions; and 3) model predictions can be used to theoretically understand the basic characteristics of the S-shaped wind profile. The practical application of the theory developed here is to provide a canopy drag coefficient profile for the numerical simulation of canopy flow. The procedures are as follows: first, the Reynolds stress profile is predicted by Eq. (27) from the observed leaf area density distribution; second, the canopy drag coefficient profile is estimated by Eq. (10) using the predicted Reynolds stress profile and observed wind profile data.

Several lines of further research on canopy flow theory, observations, and their applications are needed. First, intensive measurements of turbulent flow statistics and fluxes, net radiation, and scalars at multiple levels on existing eddy flux towers are required to further test the hypotheses. Second, the development of theories of canopy mass and energy transfer, which are similar to that of canopy momentum transfer developed in this paper, are needed. Third, the characteristics of the wind speed profile and Reynolds stress profile are different between, above, and within canopy as shown in Fig. 1. A description of the connection between the two fundamental profiles at the top of canopy or at the top of the roughness sublayer is needed. Fourth, the canopy layer is likely one of the weakest links among the entire suite of boundary layer parameterizations in global and mesoscale models since the mixing length theory (or $K$ theory) and Monin-Obukhov similarity theory are not valid within canopy. Applying the momentum transfer theory to improve the land surface parameterizations for the mesoscale models such as the Weather Research and Forecasting Model (WRF) and the Air Quality Model are encouraged. Fifth, using the analytical solutions developed here to assimilate canopy flow measurements from the eddy flux networks into large-scale numerical models is encouraged.

Acknowledgments. I am grateful for valuable comments from Drs. Dean Anderson (U.S. Geological Survey), Joshua Hacker (National Center for Atmospheric Research), Brian Lamb (Washington State University), Robert Leben (University of Colorado), William Massman (U.S. Department of Agriculture), Russell Monson (University of Colorado), Michael Novak (The University of British Columbia), Maggie Prater (University of Colorado), Jielun Sun (National Center for Atmospheric Research), Weiguo Wang (Pacific Northwest National Laboratory), and Zhiqiang Zhai (University of Colorado). 


\section{APPENDIX}

\section{Data and Measurements}

Data from observations of leaf area density and Reynolds stress, and predictions from higher-order closure models, were taken from the literature (Amiro 1990; Baldocchi and Meyers 1988; Katul and Albertson 1998; Katul et al. 2004; Kelliher et al. 1998; Shaw 1977; Wilson 1988) by digitizing published graphs when necessary. The experiments, $\mathrm{COa}$ and $\mathrm{COb}$, were conducted in two different corn canopies in Elora, Ontario, Canada, in 1971 (Wilson 1988), 1976, and 1977 (Amiro 1990; Wilson 1982). The Reynolds stress profiles of COa were measured using hot-film anemometers, while in $\mathrm{COb}$, servo-controlled split-film heat-transfer anemometers were used. The observed stress data for $\mathrm{COb}$ were mean values for each measurement level from Table 1 (Amiro 1990; Wilson 1982). The experiments for aspen (AS), jack pine (JPI), and spruce (SP) were conducted in three different boreal forest canopy sites near Whiteshell Nuclear Research Establishment in southeastern Manitoba, Canada (Amiro 1990; Wilson 1982). The Reynolds stress profiles were measured by two triaxial sonic anemometers (Applied Technology Inc., Boulder, Colorado): one operated above the forest canopy while the other was roving at different heights. The experiments for oak-hickory-pine (HW) were conducted near Oak Ridge, Tennessee (Baldocchi and Meyers 1988). The Reynolds stress profiles were measured using three simultaneous Gill sonic anemometers. The experiments for loblolly pine (LPI) were conducted at the Blackwood division of the Duke Forest near Durham, North Carolina (Katul and Albertson 1998). The Reynolds stress profiles were simultaneously measured at six levels using five Campbell Scientific CSAT3 (Campbell Scientific, Logan, Utah) triaxial sonic anemometers within the canopy and a Solent Gill sonic anemometer above the canopy. The experiments for Scots pine (SPI) were carried out at 40 $\mathrm{km}$ southwest of the village of Zotino along the western bank of the Yenisei River in central Siberia, Russia (Kelliher et al. 1998, 1999). The Reynolds stress profiles were measured using five sonic anemometers (Solent R3, Gill Instruments, Lymington, United Kingdom). All leaf area density profiles were measured across the eight sites either by destructive harvest or by plant canopy analyzers.

\section{REFERENCES}

Albini, F. A., 1981: A phenomenological model for wind speed and shear stress profiles in vegetation cover layers. J. Appl. Meteor., 20, 1325-1335.
Allen, L. H., Jr., 1968: Turbulence and wind speed spectra within a Japanese larch plantation. J. Appl. Meteor., 7, 73-78.

Amiro, B. D., 1990: Comparison of turbulence statistics within three boreal forest canopies. Bound.-Layer Meteor., 51, 99121.

Ayotte, K. W., J. J. Finnigan, and M. R. Raupach, 1999: A secondorder closure for neutrally stratified vegetative canopy flows. Bound.-Layer Meteor., 90, 189-216.

Baldocchi, D. D., and T. P. Meyers, 1988: Turbulence structure in a deciduous forest. Bound.-Layer Meteor., 43, 345-364.

Barr, S., 1971: Modeling study of several aspects of canopy flow. Mon. Wea. Rev., 99, 485-493.

Belcher, S. E., N. Jerram, and J. C. R. Hunt, 2003: Adjustment of a turbulent boundary layer to a canopy of roughness elements. J. Fluid Mech., 488, 369-398.

Bergen, J. D., 1971: Vertical profiles of windspeed in a pine stand. For. Sci., 17, 314-322.

Bohm, M., J. J. Finnigan, and M. R. Raupach, 2000: Dispersive fluxes and canopy flows: Just how important are they? Preprints, 24th Conf. on Agricultural and Forest Meteorology, Davis, CA, Amer. Meteor. Soc., 106-107.

Boussinesq, J., 1877: Theorie de l'ecoulement tourbillant. Mem. Presentés Acad. Sci. Paris, 23, 56-58.

Brunet, Y., J. J. Finnigan, and M. R. Raupach, 1994: A wind tunnel study of air flow in waving wheat: Single-point velocity statistics. Bound.-Layer Meteor., 70, 95-132.

Brunt, D., 1939: Physical and Dynamical Meteorology. 2nd ed. Cambridge University Press, 428 pp.

Cheng, H., and I. Castro, 2002: Near wall flow over urban-like roughness. Bound.-Layer Meteor., 104, 229-259.

Christen, A., and R. Vogt, 2004: Direct measurements of dispersive fluxes within a cork oak canopy. Preprints, 26th Conf. on Agricultural and Forest Meteorology, Vancouver, BC, Canada, Amer. Meteor. Soc., 23-27.

Cionco, R. M., 1965: A mathematical model for air flow in a vegetation canopy. J. Appl. Meteor., 4, 517-522.

_ 1972: A wind-profile index for canopy flow. Bound.-Layer Meteor., 3, 255-263.

Deacon, E. L., 1949: Vertical diffusion in the lowest layers of the atmosphere. Quart. J. Roy. Meteor. Soc., 75, 89-103.

Denmead, O. T., and E. F. Bradley, 1985: Flux-gradient relationships in a forest canopy. The Forest-Atmosphere Interaction, B. A. Hutchison and B. B. Hicks, Eds., D. Reidel Publishing, $421-442$.

Finnigan, J. J., 1985: Turbulence transport in flexible plant canopies. The Forest-Atmosphere Interaction, B. A. Hutchison and B. B. Hicks, Eds., D. Reidel Publishing, 443-480.

, 2000: Turbulence in plant canopies. Annu. Rev. Fluid Mech., 32, 519-571.

Fischenich, J. C., 1996: Velocity and resistance in densely vegetated floodways. Ph.D. thesis, Colorado State University, 203 pp.

Fons, R. G., 1940: Influence of forest cover on wind velocity. $J$. For., 38, 481-486.

Grant, R. H., 1983: The scaling of flow in vegetative structures. Bound.-Layer Meteor., 27, 171-184.

Inoue, E., 1963: On the turbulent structure of air flow within crop canopies. J. Meteor. Soc. Japan, 41, 317-326.

Jackson, P. S., 1981: On the displacement height in the logarithmic velocity profile. J. Fluid Mech., 111, 15-25.

Katul, G. G., and J. D. Albertson, 1998: An investigation of higher-order closure models for a forested canopy. Bound.Layer Meteor., 89, 47-74. 
— , and W. H. Chang, 1999: Principal length scales in secondorder closure models for canopy turbulence. J. Appl. Meteor., 38, 1631-1643.

—, L. Mahrt, D. Poggi, and C. Sanz, 2004: One- and twoequation models for canopy turbulence. Bound.-Layer Meteor., 113, 81-109.

Kelliher, F. M., and Coauthors, 1998: Evaporation from a central Siberian pine forest. J. Hydrol., 205, 279-296.

— , and Coauthors, 1999: Carbon dioxide efflux density from the floor of a central Siberian pine forest. Agric. For. Meteor., 94, 217-232.

Lalic, B., and D. T. Mihailovic, 2002: A new approach in parameterization of momentum transport inside and above forest canopy under neutral conditions. Integrated Assessment and Decision Support: Proc. First Biennial Meeting of the Int. Environmental Modelling and Software Society, Vol. 2, Lugano, Switzerland, IEMSS, 139-154.

Landsberg, J. J., and G. B. James, 1971: Wind profiles in plant canopies: Studies on an analytical model. J. Appl. Ecol., 8, 729-741.

— , and D. B. B. Powell, 1973: Surface exchange characteristics of leaves subject to mutual interference. Agric. For. Meteor., 12, 169-184.

Legg, B. J., and I. F. Long, 1975: Turbulent diffusion within a wheat canopy: II. Results and interpretation. Quart. J. Roy. Meteor. Soc., 101, 611-628.

Lemon, E., L. H. Allen, and L. Muller, 1970: Carbon dioxide exchange of a tropical rain forest. 2. BioScience, 20, 1054-1059.

Macdonald, R. W., 2000: Modelling the mean velocity profile in the urban canopy layer. Bound.-Layer Meteor., 97, 25-45.

Maheshwari, B. L., 1992: Suitability of different flow equations and hydraulic resistance parameters for flows in surface irrigation: A review. Water Resour. Res., 28, 2059-2066.

Mahrt, L., X. Lee, A. Black, H. Neumann, and R. M. Staebler, 2000: Nocturnal mixing in a forest subcanopy. Agric. For. Meteor., 101, 67-78.

—, D. Vickers, J. L. Sun, N. O. Jensen, H. Jorgensen, E. Pardyjak, and H. Fernando, 2001: Determination of the surface drag coefficient. Bound.-Layer Meteor., 99, 249-276.

Massman, W. J., 1987: A comparative study of some mathematical models of the mean wind structure and aerodynamic drag of plant canopies. Bound.-Layer Meteor., 40, 179-197.

_ 1997: An analytical one-dimensional model of momentum transfer by vegetation of arbitrary structure. Bound.-Layer Meteor., 83, 407-421.

_ order closure model of turbulence statistics and the Lagrangian time scale within and above plant canopies of arbitrary structure. Bound.-Layer Meteor., 91, 81-107.

Meyers, T., and K. T. Paw U, 1986: Testing of a higher-order closure model for modeling airflow within and above plant canopies. Bound.-Layer Meteor., 37, 297-311.

Mohan, M., and M. K. Tiwari, 2004: Study of momentum transfer within a vegetation canopy. Proc. Indian Acad. Sci., Earth Planet. Sci., 113, 67-72.

Monteith, J. L., and M. H. Unsworth, 1990: Principles of Environmental Physics. 2nd ed. Edward Arnold, 291 pp.

Murota, A., T. Fukuhara, and M. Sato, 1984: Turbulence structure in vegetated open channel flows. J. Hydrosci. Hydraul. Eng., 2, 47-61.

Novak, M. D., J. S. Warland, A. L. Orchansky, R. Ketler, and S. Green, 2000: Wind tunnel and field measurements of turbu- lent flow in forests. Part I: Uniformly thinned stands. Bound.Layer Meteor., 95, 457-495.

Obukhov, A. M., and A. S. Monin, 1953: Dimensionless characteristics of turbulence in the atmospheric surface layer. Dokl. Akad. Nauk SSSR, 93, 223-226.

Oke, T. R., 1987: Boundary Layer Climates. 2nd ed. Methuen, 435 pp.

Oliver, H. R., 1971: Wind profiles in and above a forest canopy. Quart. J. Roy. Meteor. Soc., 97, 548.

Pinard, J., and J. D. Wilson, 2001: First- and second-order closure models for wind in a plant canopy. J. Appl. Meteor., 40, 17621768.

Poggi, D., A. Porporato, L. Ridolfi, J. D. Albertson, and G. G. Katul, 2004a: The effect of vegetation density on canopy sublayer turbulence. Bound.-Layer Meteor., 111, 565-587.

—, G. G. Katul, and J. D. Albertson, 2004b: A note on the contribution of dispersive fluxes to momentum transfer within canopies. Bound.-Layer Meteor., 111, 615-621.

Prandtl, L., 1925: Uber die ausgebildete turbulenz. Z. Angew. Math. Mech., 5, 136-139.

Raupach, M. R., 1992: Drag and drag partition on rough surfaces. Bound.-Layer Meteor., 60, 375-395.

- and A. S. Thom, 1981: Turbulence in and above plant canopies. Annu. Rev. Fluid Mech., 13, 97-129.

, and R. H. Shaw, 1982: Averaging procedures for flow within vegetation canopies. Bound.-Layer Meteor., 22, 79-90.

—, P. A. Coppin, and B. J. Legg, 1986: Experiments on scalar dispersion within a model plant canopy. Part I: The turbulence structure. Bound.-Layer Meteor., 35, 21-52.

Schimel, D. S., and Coauthors, 2001: Recent patterns and mechanisms of carbon exchange by terrestrial ecosystems. Nature, 414, 169-172.

Schlichting, H., 1960: Boundary Layer Theory. 4th ed. McGrawHill, 647 pp.

Schuepp, P. H., 1984: Observations on the use of analytical and numerical models for the description of transfer to porous surface vegetation such as lichen. Bound.-Layer Meteor., 29, $59-73$.

Shaw, R. H., 1977: Secondary wind speed maxima inside plant canopies. J. Appl. Meteor., 16, 514-521.

Stull, R. B., 1988: An Introduction to Boundary Layer Meteorology. Kluwer Academic, 670 pp.

Sutcliffe, R. C., 1936: Surface resistance in atmospheric flow. Quart. J. Roy. Meteor. Soc., 62, 3-12.

Sutton, O. G., 1953: Micrometeorology: A Study of Physical Processes in the Lowest Layers of the Earth's Atmosphere. McGraw-Hill, 333 pp.

Taylor, G. I., 1916: Skin friction of the wind on the earth's surface. Proc. Roy. Soc. London, 92, 196-199.

Thom, A. S., 1968: Exchange of momentum, mass, and heat between an artificial leaf and the airflow in a wind-tunnel. Quart. J. Roy. Meteor. Soc., 94, 44-55.

_ 1971: Momentum absorption by vegetation. Quart. J. Roy. Meteor. Soc., 97, 414.

Turnipseed, A. A., D. E. Anderson, P. D. Blanken, W. M. Baugh, and R. K. Monson, 2003: Airflows and turbulent flux measurements in mountainous terrain. Part 1: Canopy and local effects. Agric. For. Meteor., 119, 1-21.

Uchijima, Z., and J. Wright, 1964: An experimental study of air flow in a corn plant-air layer. Bull. Natl. Inst. Agric. Sci., Ser. A, 11, 19-66.

Vitousek, P. M., J. D. Aber, R. W. Howarth, G. E. Likens, P. A. Matson, D. W. Schindler, W. H. Schlesinger, and D. G. Til- 
man, 1997: Human alteration of the global nitrogen cycle: Sources and consequences. Ecol. Appl., 7, 737-750.

von Kármán, T., 1930: Mechanische ähnlichkeit and yurbulenz. Nachr. Ges. Wiss. Göettingen Math. Phys. Kl., 68, 58-76.

Wilson, J. D., 1982: Turbulent dispersion in the atmospheric surface layer. Bound.-Layer Meteor., 22, 399-420.

_ 1988: A 2nd-order closure model for flow through vegetation. Bound.-Layer Meteor., 42, 371-392.

Wilson, N. R., and R. H. Shaw, 1977: Higher-order closure model for canopy flow. J. Appl. Meteor., 16, 1197-1205.

Wofsy, S. C., 2001: Where has all the carbon gone? Science, 292, 2261-2263.
Wyngaard, J. L., 1973: On surface-layer turbulence. Workshop on Micrometeorology, D. A. Haugen, Ed., Amer. Meteor. Soc., 101-149.

Yi, C., R. K. Monson, Z. Zhai, D. E. Anderson, B. Lamb, G. Allwine, A. A. Turnipseed, and S. P. Burns, 2005: Modeling and measuring the nocturnal drainage flow in a high-elevation, subalpine forest with complex terrain. J. Geophys. Res., 110, D22303, doi:10.1029/2005JD006282.

, D. E. Anderson, A. A. Turnipseed, S. P. Burns, J. Sparks, D. Stannard, and K. R. Monson, 2008: The contribution of advective fluxes to net ecosystem $\mathrm{CO}_{2}$ exchange in a high-elevation, subalpine forest ecosystem. Ecol. Appl., in press. 